\title{
Circularly polarized undulator radiation from the new double crossed undulator beamline at BESSY and its first use for spin resolved Auger electron emission spectroscopy
}

\author{
R. David *, P. Stoppmanns, S.-W. Yu, R. Kuntze, N. Müller, U. Heinzmann \\ Unversität Bielefeld, Fak. für Physik, D-33501 Btelefeld, Germany and Fritz-Haber-Institut der MPG, D-14195 Berlin, Germany
}

(Received 25 November 1993)

\begin{abstract}
The new beamline U2-FSGM at BESSY provides now circularly polarized radiation of high flux for photon energies between 20 $\mathrm{eV}$ and $200 \mathrm{eV}$. For users working at this beamline it is essential to measure the photon polarization. We have developed a new apparatus consisting of a Rabinovitch detector for analyzing the photon polarization, a $90^{\circ}$ spherical electron spectrometer and a small retarding spherıcal Mott polarimeter $(45 \mathrm{keV})$ for determining the electron spin polarizatıon. As a first application we have measured the $\mathrm{M}_{3} \mathrm{VV}$ Auger emission from $\mathrm{Cu}(100)$ spin resolved after photo excitation by means of circularly polarized radiation of $76 \mathrm{eV}$.
\end{abstract}

\section{Introduction}

The new U2-FSGM beamline at BESSY is a high flux source of elliptically polarized radiation equipped with a high resolution focussed spherical grating monochromator for photon energies up to $200 \mathrm{eV}[1,2]$. Now investigations with circularly polarized radiation at energies higher than the normal incidence range are possible which need a high photon flux or a small bandwidth of the radiation, e.g. spin resolved Auger spectroscopy after excitation of oriented core hole states. It is the aim of this paper to present first experiences and results in the use of this beamline from a first user point of view. Essential for working at this beamline is the use of an analyzer for characterizing the photon polarization, in our experiment a Rabinovitch polarimeter [3,4]. In our new apparatus for working at the U2-FSGM, consisting also of a $90^{\circ}$ spherical electron spectrometer and a small retarding spherical Mott polarimeter [5], the Rabinovitch polarimeter is located behind the target crystal which can be removed for undulator radiation analysis. In the following we briefly report on the properties of the crossed double undulator and the monochromator, and we describe our Rabinovitch detector and its feasibility for analyzing the radiation and we portray our electron spectrometer with the Mott polarimeter. As a documentation of the feasibility of the whole setup we show

\footnotetext{
* Corresponding author.
}

first spin resolved results for the $\mathrm{M}_{3} \mathrm{VV}$ Auger electrons from $\mathrm{Cu}(100)$ following photo excitation with circularly polarized radiation of $76 \mathrm{eV}$.

\section{Undulator and monochromator}

The crossed double undulator at BESSY [1] consists of two undulators in series according to the proposal of Kim [6]. They are rotated against each other by an angle of $90^{\circ}$. Each of the undulators gives linearly polarized radiation. This is partly coherently superimposed in the monochromator by diffraction at the grating. Depending on the phase difference between the two waves coherently superimposed and their intensities, the radiation is partly linearly under $45^{\circ}$, elliptically or circularly polarized. By working with only one undulator a vertical or horizontal linearly polarized radiation is obtained. To change the phase difference of the waves coming from each undulator, i.e. to change the state of polarization, a modulator is inserted between the two undulators. Its magnetic field is excited electrically. Therefore the phase difference can be scanned by changing the modulator current. In this way the polarization of the radiation can be varied from linear to circular or from $\sigma^{+}$to $\sigma^{-}$. Using this phase shifting it is possible to reveal the total degree of polarization by using a polarimeter which is only sensitive to linear polarization and cannot in principle distinguish between unpolarized and circularly polarized radiation, e.g. a Rabinovitch polarimeter [3,4]. The 
maximum degree of circular polarization at a certain modulator current is equivalent to the measured maximum degree of linear polarization at another modulator current, under the assumption that the partial coherent superposition of the two waves from both undulators and thus the total degree of polarization does not change with the modulator current.

A movable pinhole limits the lightbeam to $2 \mathrm{~mm}$ width in front of the monochromator. The Focussed Spherical Grating Monochromator (FSGM) [2] uses an entrance slit of variable width which is $5-3000 \mu \mathrm{m}$ wide. In practice the upper usable limit is $200 \mu \mathrm{m}$ only [7] which is the image of the undulator radiation source. The entrance slit is imaged horizontally one to one by mirrors and a grating onto the 5-3000 $\mu \mathrm{m}$ wide exit slit, which is again imaged one to one by an additional mirror onto the target. For a high intensity in our experiment we chose $100 \mu \mathrm{m}$ and $1200 \mu \mathrm{m}$ for the entrance and exit slit, respectively, because we did not need the extreme resolution of the FSGM. As practical experience for the users of this beamline it is worth noting that the photon beam position on the target is highly stable since it is the image of the exit slit. The settings of the beamline have been controlled by measuring the intensity of the radiation on a Au net which can be inserted behind the exit slit.

\section{The light polarimeter}

To measure the photon polarization at the U2FSGM we have built up a Rabinovitch polarimeter $[3,4]$. Besides the setup as a whole Fig. 1 also contains a scheme of the polarimeter. The incoming light is impinging on a Si wafer under the angle of $45^{\circ}$ with the consequence that at this angle the reflectivity for $\mathrm{s}$ - and p-polarized light generally follows the relationship $R_{\mathrm{s}}^{2}$ $=R_{\mathrm{p}}$ [3]. From the Henke-data [8] for the reflectivity of $\mathrm{Si}$ it follows that the reflected light in the energy range used here is almost linearly polarized and thus the $\mathrm{Si}$ wafer acts as a linear polarizer. The reflected beam hits a $\mathrm{Au}$ photodiode realized by a $\mathrm{Au}$ film evaporated onto a glass disc. The photo current itself is in the $10^{-10} \mathrm{~A}$ range. Due to the Si $\mathrm{L}$ edge the Si wafer is usable for photon energies up to approximately 100 $\mathrm{eV}$, for higher photon energies it has to be changed to a Au mirror. The Rabinovitch polarimeter as a linear analyzer cannot distinguish between unpolarized and circularly polarized radiation by itself. But the technique of systematic phase variation of the wavetrains coming from each undulator gives an alternative discussed above. Fig. 2 shows a set of rotation diagrams measured with the Rabinovitch polarimeter for different modulator currents for the photon energy $h \nu=43$ $\mathrm{eV}$. In these diagrams the rotation angle $\phi$ refers to the horizontal direction of the first undulator. By increasing the modulator current, the phase shift increases continuously, yielding linearly polarized radiation, circularly polarized radiation, again linearly polarized radiation $\left(90^{\circ}\right.$ rotated), again circularly polarized radiation (of the other helicity) and so on with a constant unpolarized background. Fig. 3 demonstrates this by showing the degree of linear polarization of the partially elliptical radiation. Maxima of the linear polarization where there is a vanishing circular component are present at $25 \mathrm{~A} \pm 0.5 \mathrm{~A}$ and at $32 \mathrm{~A} \pm 0.5 \mathrm{~A}$. The minima of the linear polarization where the light is circularly polarized (besides the unpolarized back-

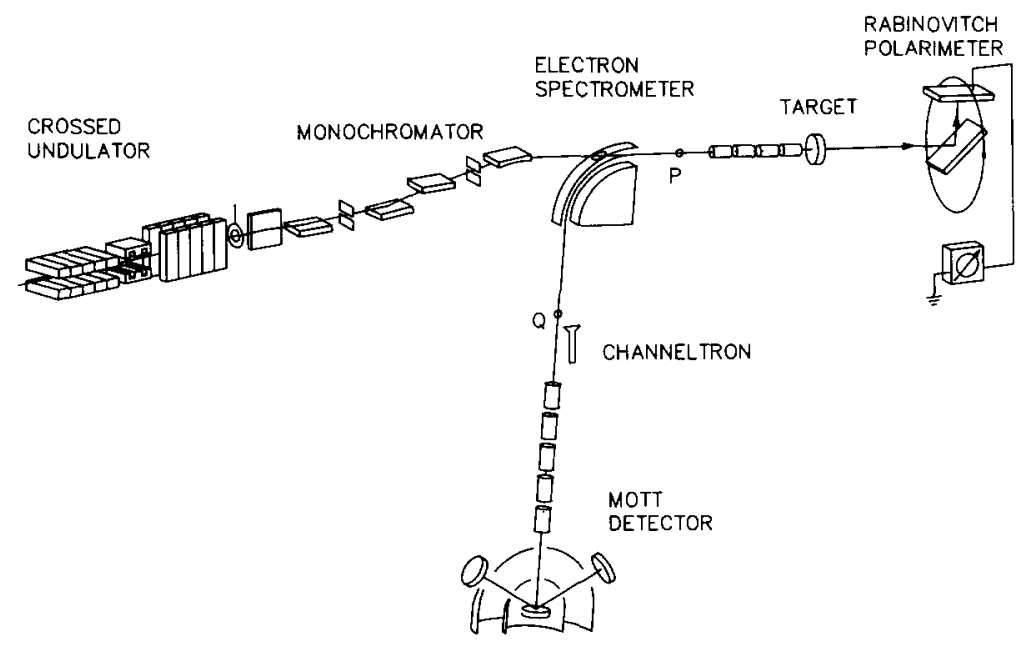

Fig. 1. Experimental setup. The distances are not to scale. The target can be removed from the light beam for measurements with the Rabinovitch polarimeter. For details of the setup see text. 


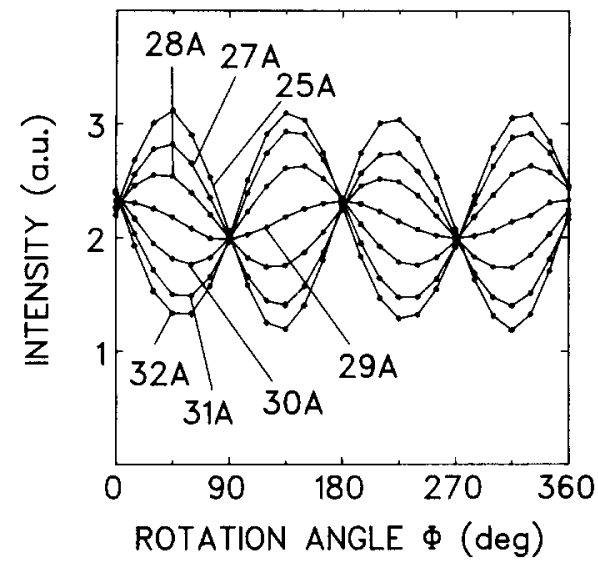

Fig. 2. Rotation diagrams of the Rabinovitch polarimeter for different modulator currents for $43 \mathrm{eV}$ photon energy. The direction of $\phi=0^{\circ}$ is given by the horizontal direction of the first undulator.

ground) are present at $21 \mathrm{~A} \pm 0.5 \mathrm{~A}$ and $29 \mathrm{~A} \pm 0.5 \mathrm{~A}$. the phaseshift revealed in changing the modulator current from $25 \mathrm{~A}$ to $32 \mathrm{~A}$ or from $21 \mathrm{~A}$ to $29 \mathrm{~A}$ corresponds to half of the wavelength of the radiation. The fact that the peak heights in Fig. 3 do not change when going from $17 \mathrm{~A}$ to $25 \mathrm{~A}$ to $32 \mathrm{~A}$ within the experimental uncertainty is the evidence that the total degree of polarization and thus the degree of coherence of the two waves leaving both undulators does not change with the modulator current. Thus this total degree of polarization is the degree of circular polarization, when the linear polarization has its minima at $21 \mathrm{~A}$ and $29 \mathrm{~A}$. Fig. 4 shows the total polarization, i.c. the circular polarization obtainable as a function of the photon energy. Open circles are measurements in the

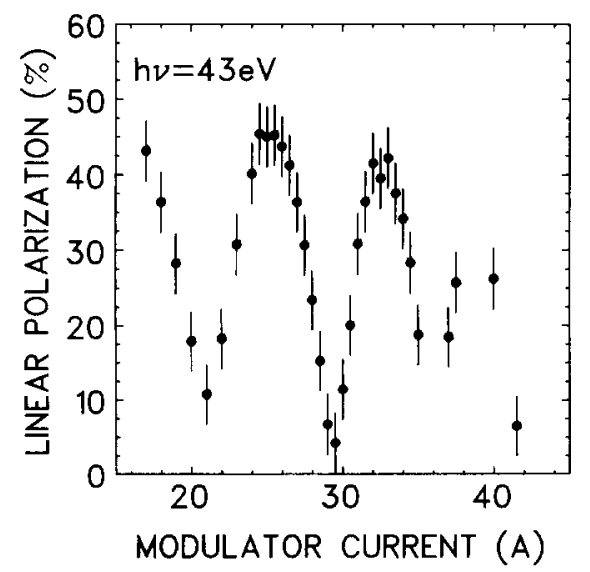

Fig. 3. Linear polarization versus modulator current for a photon energy of $43 \mathrm{eV}$. The error bars include statistical and systematical errors.

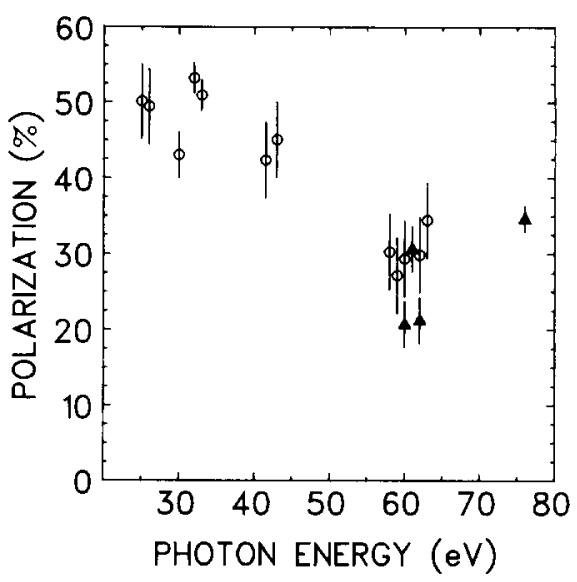

Fig. 4. Degree of polarization versus photon energy Open circles are measured in the first harmonic of the radiation, filled triangles are measured in the third harmonic of the radiation. The error bars include statistical and systematical errors.

first harmonic of the radiation, filled triangles are measurements in the third harmonic of the radiation. An increase of the photon energy leads to a decrease of the polarization. For photon energies around $30 \mathrm{eV}$ the polarization reaches about $50 \%$ while the polarization decreases to somewhat more than $30 \%$ at photon energies above $60 \mathrm{eV}$. It is worth noting that the absolute value of the polarization reached is very sensitive to the adjustment of the undulator and the monochromator. It can easily be decreased by a factor of 1.5 , but for a chosen setting it is stable.

\section{Experimental setup for spin resolved electron spec- troscopy}

The apparatus for performing the spin resolved electron spectroscopy contains an electrostatic $90^{\circ}$ spherical electron spectrometer with a mean radius of $84 \mathrm{~mm}$ and a retarding spherical Mott polarimeter. A scheme of the experimental setup is given in Fig. 1. The construction is a normal incidence, normal emission setup. The incoming circularly polarized light passes through a hole in the spectrometer and normally hits the crystal mounted on a target manipulator. The electrons emitted inside a cone of approximately $4^{\circ}$ around the surface normal are accelerated to the pass energy of $100 \mathrm{eV}$ and imaged onto the virtual entrance slit $\mathrm{P}$ of the $90^{\circ}$ spherical spectrometer by a four element electrostatic zoom lens [9]. The spectrometer images the virtual entrance slit $P$ to the exit slit at $\mathrm{Q}$ opened to result in a $1 \%$ energy resolution. Intensity spectra without spin polarization analysis are effec- 
tively measured by means of a flat ceramic channeltron [10] behind a second exit slit near to the central trajectory of the electrons. The Auger electron intensity detected there has been found to be so high that the channeltron must be operated at about $1 / 10$ of its maximum sensitivity to prevent saturation effects at count rates $>10^{5}$ counts $/ \mathrm{s}$.

After passing the exit slit at $Q$ the electrons are imaged onto the entrance of a retarding spherical field Mott polarimeter [5] of small size by a multiple lens system including two four-pole deflectors for alignment of the electron beam. The inner sphere and outer sphere of the Mott polarimeter have the radii $23.5 \mathrm{~mm}$ and $47 \mathrm{~mm}$, respectively. The spheres are inside a vacuum housing which consists of a halfsphere with radius $100 \mathrm{~mm}$ and a $160 \mathrm{~mm}$ diameter tube containing the inverse mounted high voltage feedthroughs. There is no unshielded high voltage present. In the spherical field of the Mott polarimeter the electrons are accelerated to $45 \mathrm{keV}$ by the spherical field and then scattered at a $0.2 \mathrm{~mm}$ thick $\mathrm{Au}$ sheet in the center of the spheres. Electrons scattered inside a cone of $\pm 11^{\circ}$ around the $\pm 120^{\circ}$ directions enter again the spherical field and are now decelerated. Only electrons with inelastic energy losses of less than $700 \mathrm{eV}$ will penetrate through the retarding meshes in front of two pairs of microchannelplates and will be counted. Typical count rates in the Mott polarimeter are 300 counts/s-500 counts/s at a background of $<5$ counts $/ \mathrm{s}$ in the $\mathrm{Cu}(100) \mathrm{M}_{3} \mathrm{VV}$ Auger peak, excited with radiation of $76 \mathrm{eV}$ at a BESSY ring current of 500 $\mathrm{mA}$. The channelplates, however, are not operated at maximum efficiency to prevent noise instabilities.

The spherical Mott polarimeter is positioned such that the component of the spin polarization vector $\boldsymbol{P}$ parallel to the incoming light beam is measured. The helicity of the radiation is changed to eliminate apparatus asymmetries after every scan through the Auger electron spectrum, i.e. every $15 \mathrm{~min}$. The spin polarization is determined from the left/right scattering asymmetry using the polarization sensitivity $S_{\text {eff }}=0.18 \pm$ $0.02[11]$.

\section{Experiment}

For a first application of the whole setup described we have measured the spin polarization across the $\mathrm{Cu}(100) \mathrm{M}_{3}$ VV Auger emission peak after excitation of an oriented primary hole by partly circularly polarized radiation of $76 \mathrm{eV}$ photon energy. The degree of circular polarization of the radiation was $(34 \pm 1.7) \%$. The $\mathrm{Cu}$ target was cleaned by cycles of $\mathrm{Ar}^{+}$and $\mathrm{Ne}^{+}$ ion bombardment and annealing up to $400^{\circ} \mathrm{C}$ [12]. The photon energy used can only create $3 \mathrm{p}_{3 / 2}$ holes, since

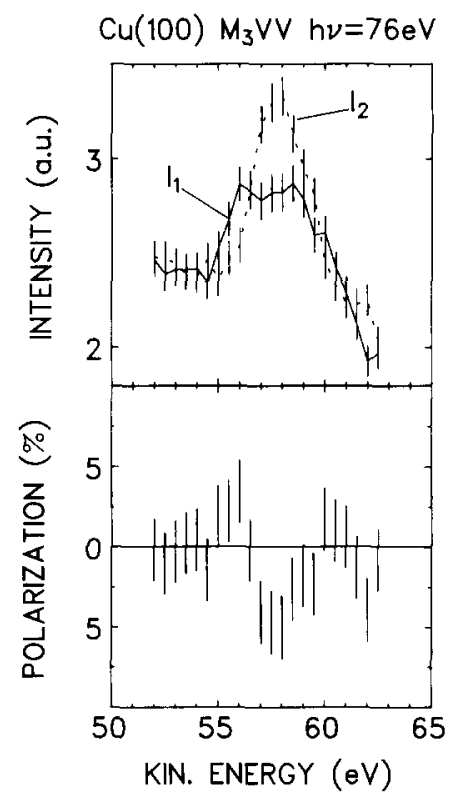

Fig. 5. Upper panel: spin separated $\mathrm{M}_{3} \mathrm{VV}$ Auger spectrum including the inelastic background after excitation with circularly polarized radiation of $76 \mathrm{eV}$. The error bars include the statistical error and the errors of $S_{\text {eff }}$ and $P_{\text {crk }}$. For $I_{1}$ and $I_{2}$ see text. Lower panel: spin polarization of the Auger peak versus kinetic energy normalized to a $100 \%$ circularly polarized radiation but without corrections with respect to the unpolarized background of the peak in the upper panel. The measurements do not give the sign of the spin polarization. The error bars include the statistical error and the errors of $S_{\text {efr }}$ and $P_{\text {clrk }}$.

the binding energy for the $3 p_{3 / 2}$ and the $3 p_{1 / 2}$ electron is $75.2 \mathrm{eV}$ and $77.2 \mathrm{eV}$, respectively [13]. The valence bands are dominated by $d$-like states centered about 3 $\mathrm{eV}$ below $E_{\mathrm{F}}[14,15]$. The $3 \mathrm{p}_{3 / 2}$ hole is filled by an electron from the valence band under emission of a second valence band electron as the Auger electron. The kinetic energy of the Auger electron is about 58 $\mathrm{eV}$ due to a two hole correlation energy of about 7.7 $\mathrm{eV}$ [13]. Fig. 5 shows the spin separated partial intensities $I_{1}$ and $I_{2}$ (upper panel) and the corresponding spin polarization (lower panel) measured across the $\mathrm{Cu}(100) \mathrm{M}_{3} \mathrm{VV}$ Auger peak; spin polarization and partial intensities are connected by $P=\left(I_{1}-I_{2}\right) /\left(I_{1}+I_{2}\right)$.

A significant spin polarization is present at the center of the Auger peak. Towards the edges of the peak the sign of the spin polarization changes. It is worth noting that the data shown in Fig. 5 are normalized to a $100 \%$ degree of circular polarization but not corrected with respect to the unpolarized background of the peak in Fig. 5, upper panel. Unfortunately the absolute sign of the spin polarization is still not known, since the sign of the photon helicity of the radiation 
has not yet been measured absolutely. This has to be done in the future by measuring the spin polarization of photoelectrons at a photon energy in the normal incidence range where reference data are available from the experiment at the $6.5 \mathrm{~m}$ NIM monochromator [16].

\section{Conclusion}

The new crossed undulator U2 with the monochromator FSGM opens a field for new investigations using circularly polarized radiation at high flux. It is essential for using the circularly polarized radiation to control the state of light polarization. We showed that a Rabinovitch polarimeter is a usable tool for analyzing the radiation. In a first application on the U2-FSGM we have given an example to show that this new beamline is suitable for spin resolved measurements of $\mathrm{CVV}$ Auger electrons after excitation with circularly polarized radiation.

\section{Acknowledgement}

We thank the BESSY staff, especially A. Gaupp, G. Reichardt, J. Bahrdt and W.B. Peatman for their support at the new beamline and for fruitful discussions. We also give thanks to N. Böwering and Th. Döhring for support in developing the Rabinovitch polarimeter. This work is supported by the "Bundesministerium für Forschung und Technologie" No. 055PBAXI3.

\section{References}

[1] J. Bahrdt, A. Gaupp, W. Gudat, M. Mast, K. Molter, W.B. Peatman, M. Scheer, Th. Schroeter and Ch. Wang, Rev. Sci. Instr. 63 (1992) 339.

[2] W.B. Peatman, J. Bahrdt, F. Eggenstein and F. Senf, BESSY Annual Report (1991) p. 471.

[3] K. Rabinovitch, L.R. Canfield and R.P. Madden, Appl. Opt. 4 (1963) 1005.

[4] J.A.R. Samson, Rev. Sci. Instr. 47 (1976) 859.

[5] L.G. Gray, M.W. Hart, F.B. Dunning and G.K. Walters, Rev. Sci. Instr. 55 (1984) 88.

[6] K.J. Kim, Nucl. Instr. and Meth. 222 (1984) 11.

[7] G. Reichardt, private communication.

[8] B.L. Henke, P. Lee, T.J. Tanaka, R.L. Shimabukuro and B.K. Fujikawa, At. Data Nucl. Data Tables 27 (1982) 1.

[9] G. Martinez, M. Sancho and F.H. Read, J. Phys E 16 (1983) 631.

[10] KBL 110, sensitive entrance $1 \mathrm{~mm} \times 10 \mathrm{~mm}$, Dr. Suits, D-24568 Kaltenkirchen, Germany.

[11] J. Kessler, Polarized Electrons, 2nd ed. (Springer, Berlin, 1985).

[12] R.G. Musket, W. McLean, C.A. Colmanares, D.M. Makowiecki and W.J. Siekhaus, Appl. Surf. Sci. 10 (1982) 143.

[13] E. Antonides, E.C. Janse and G.A. Samwatzky, Phys. Rev. B 15 (1977) 1669.

[14] D.A. Papaconstantopoulos, Handbook of the Band Structure of Elemental Solıds (Plenum, New York, 1986).

[15] H. Eckardt, L. Fritsche and J. Noffke, J. Phys. F 14 (1984) 97.

[16] A. Eyers, F. Schäfers, G. Schönhense, U. Heinzmann, H.P. Oepen, K. Hünlich, J. Kirschner and G. Borstel, Phys. Rev. Lett. 52 (1984) 1559. 